\title{
Thymidine to Cytosine Transition Abnormality
}

National Cancer Institute

\section{Source}

National Cancer Institute. Thymidine to Cytosine Transition Abnormality. NCI Thesaurus. Code C45642.

A point mutation involving the substitution of Cytosine (a pyrimidine base) for Thymidine (a pyrimidine base) in a DNA sequence from eukaryotic or prokaryotic organisms. This abnormality can be either heritable or occur somatically. 